\title{
Invasive and Noninvasive Correlations of B-Type Natriuretic Peptide in Patients With Heart Failure Due to Chagas Cardiomyopathy
}

$\mathrm{H}^{\mathrm{n}}$ eart failure is becoming a major health problem in both developing and developed countries. ${ }^{1}$ In Latin America, this problem is aggravated by the presence of almost 11 million persons with Chagas heart disease. ${ }^{2}$ Because of intense immigration from endemic areas, transfusion-related infection is now viewed as a potential threat in the United States. ${ }^{3}$ Chronic Chagas cardiomyopathy is a model of heart failure that is characterized by persistent diffuse myocarditis, in which a cross-reactive autoimmunity component is responsible for the maintenance of cellular aggression. ${ }^{4,5}$ The heart is affected throughout, and patients may present signs and symptoms of both right and left heart failure, which has important prognostic value. ${ }^{6}$ Because of right ventricular compromise, the volume status of patients with Chagas disease heart failure may be difficult to evaluate, leading to potential overuse of diuretics. No specific treatment is available, although recent data indicate that etiologic treatment may offer some benefit. ${ }^{7,8}$

Over the past few years, B-type natriuretic peptide (BNP) analysis has become an important tool in the diagnosis and risk stratification of patients with heart failure. ${ }^{9,10}$ Testing for BNP has been shown to be a cost-effective diagnostic tool in patients with heart failure, a relevant issue in developing countries. ${ }^{11}$ BNP is a neurohormone secreted mainly in heart ventricles in response to volume and pressure overload. ${ }^{12}$ Because the left ventricle

Heart failure due to Chagas cardiomyopathy (HFCC) differs from failure with other etiologies because of the occurrence of intense inflammatory infiltrate and right ventricle compromise. This article investigates correlations of B-type natriuretic peptide (BNP) levels with parameters of severity in HFCC. Twenty-eight patients and 8 normal controls underwent heart catheterization and clinical and laboratory analyses. BNP levels were higher in patients with HFCC $(\mathrm{P}<.0001)$ and correlated with New York Heart Association (NYHA) class; right atrial pressure; wedge pressure; cardiac output; levels of serum sodium, hemoglobin, urea, and tumor necrosis factor- $\alpha$; and ejection fraction. Interferon- $\gamma$ and transforming growth factor- $\beta$ did not correlate with BNP level. The authors conclude that BNP levels are elevated in patients experiencing HFCC, irrespective of NYHA class, and that the occurrence of HFCC correlates with severity of disease. (Congest Heart Fail. 2008; 14:121-126) ๑2008 Le Jacq

Fábio Vilas-Boas, MD, ScD; Gilson Soares Feitosa, MD; Milena B.P. Soares, PhD; ${ }^{2}$ Joel Alves Pinho-Filho, MD; Thais Nascimento, MD; ${ }^{1}$ Marcos M. Barojas, MD;1 Marcus V.S. Andrade, MD; Ricardo Ribeiro-dos-Santos, MD, PhD;2 Edimar Bocchi, MD, PhD

From the Heart Failure Clinics and Academic Research Department of Santa Izabel Hospital, Salvador, BA, Brazil;' Gonçalo Moniz Research Center Oswaldo Cruz Foundation, Salvador, BA, Brazil;' the Heart Institute of the University of São Paulo, SP, Brazil ${ }^{3}$

Address for correspondence:

Fábio Vilas-Boas, MD, ScD, the Heart Failure Clinics and Academic Research Department of Santa Izabel Hospital, Praça Almeida Couto, 500. Salvador, Bahia, Brazil, 40.050-510

E-mail: fabiovboas@cardiol.br.

Manuscript received January 10, 2007; revised November 27, 2007; accepted November 30, 2007

has a higher muscle mass than the right ventricle, most BNP elevations are thought to be due to left ventricle BNP secretion, rather than right ventricle overload.

Only recently, BNP testing has been introduced as a clinical tool in South America. ${ }^{13}$ Few reports on evaluation of BNP levels in patients with Chagas disease heart failure have been published, however. The available data indicate that patients with positive serologic results but no heart compromise have normal BNP levels. ${ }^{14}$ Another report has indicated that BNP levels are higher in patients with Chagas disease accompanied by apical aneurysm and complex ventricular arrhythmia than in those with apical aneurysm alone. ${ }^{15}$

Because Chagas cardiomyopathy appears to affect the entire heart, 
Table I. Demographics and Medications of Studied Population

\begin{tabular}{|lccc|}
\hline & $\begin{array}{c}\text { Heart Fallure } \\
(\mathrm{N}=28)\end{array}$ & $\begin{array}{c}\text { CONTROL } \\
(\mathrm{N}=8)\end{array}$ & $P$ VAlUe \\
\hline Age, y & $51.3 \pm 1.6$ & $53.3 \pm 2.3$ & .62 \\
Male sex, no. (\%) & $20(71.4)$ & $6(75.0)$ & .94 \\
Weight, kg & $61.2 \pm 2.1$ & $61.5 \pm 3.7$ & .98 \\
BMl, kg/m² & $22.5 \pm 0.7$ & $22.3 \pm 1.3$ & .95 \\
MAP, mm Hg & $79 \pm 12$ & $84 \pm 18$ & .32 \\
Heart rate, beats per minute & $72 \pm 11$ & $75 \pm 14$ & .54 \\
Digoxin, mg/d (no.) & $0.23 \pm 0.01(24)$ & - & - \\
Furosemide, mg/d (no.) & $67.7 \pm 6.2(26)$ & - & - \\
Hydrochlorothiazide, mg/d (no.) & $34.4 \pm 4.4(4)$ & - & - \\
Captopril, mg/d (no.) & $52.3 \pm 4.7(11)$ & - & - \\
Enalapril, mg/d (no.) & $18.2 \pm 2.2(14)$ & - & - \\
Losartan, mg/d (no.) & $41.6 \pm 8.3(3)$ & - & - \\
Spironolactone, mg/d (no.) & $25 \pm 0(22)$ & - & - \\
Carvedilol, mg/d (no.) & $32.5 \pm 7.5(5)$ & - & - \\
\hline Abbreviations: BMl, body mass & & - & - \\
\hline
\end{tabular}

Abbreviations: $\mathrm{BMI}$, body mass index; MAP, mean arterial pressure. Values presented as mean \pm SE.

we sought to investigate correlations of BNP levels with invasive hemodynamic, clinical, biochemical, and immunologic parameters of heart failure severity. Achieving a better understanding of its profile can help define the role of this new biomarker in the diagnosis and management of Chagas disease heart failure.

\section{Methods}

Participants. We prospectively studied 28 patients with chronic heart failure due to Chagas cardiomyopathy (mean age, $51.3 \pm 1.6$ years) and 8 healthy volunteers (mean age, $53.3 \pm 2.3$ years; $P=.62$ ). Twenty-two stable patients were recruited from outpatient clinics, and 6 patients with moderate or severe edema, identified on attendance to the clinic or admission to the ward, were included to represent decompensated patients. The healthy volunteers were patients who were referred for heart catheterization for medical reasons, did not have coronary artery disease or structural heart disease, and agreed to participate. That Chagas disease was the cause of chronic heart failure was confirmed by 2 serologic methods, indirect hemagglutination and indirect immunofluorescence. The diagnosis of chronic heart failure was based on symptoms arising during exercise, the presence of cardiomegaly, and docu- mented left ventricle dysfunction (all patients had a left ventricle ejection fraction [LVEF] $<40 \%$, measured by echocardiography - Simpson's rule or radionuclide ventriculography). No patient or volunteer had clinical signs of infection, lung disease, cancer, or other clinically relevant comorbidities. The research protocol was approved by the ethics committee of our institution, and all patients and volunteers gave written informed consent.

Routine Clinical Assessment. All patients and controls underwent an initial clinical assessment that, in general, included a clinical history, a physical examination, electrocardiography, routine blood tests, and chest radiography. Doppler echocardiography (Model 2500; Hewlett-Packard, Seattle, WA) was performed according to the criteria of the American Society of Echocardiography. ${ }^{16}$ The LVEF was obtained via Simpson's rule with the software provided with the equipment by an observer blinded to the clinical status. ${ }^{17}$

Quality of life was assessed by the Minnesota Living With Heart Failure Questionnaire, which was administered by the same research nurse to all participants. ${ }^{18}$ A 6-minute walking test was performed according to the protocol of the Studies of Left Ventricular Dysfunction (SOLVD). ${ }^{19,20}$
Heart Catheterization. After an 8-hour fast, patients underwent right and left heart catheterization on digital equipment (Model H-5000; Philips Medical Systems, Andover, MA). Right heart catheterization was done by brachial vein dissection, and a Lehman catheter was introduced to measure pressures. Left heart catheterization was performed by brachial artery dissection through which a Sones catheter was introduced and advanced into the aorta and left heart chambers, where the intracavitary pressures were registered and blood samples collected.

Assays. Blood samples were collected after at least a 15-minute rest. A polyethylene Lehman catheter was inserted into the brachial artery and advanced into the thoracic aorta where $10 \mathrm{~mL}$ of blood was drawn into chilled tubes containing $1.5 \mathrm{mg} / \mathrm{mL}$ 2-natrium-ethylenediamine tetra-acetic acid, kept in ice, and immediately taken to the laboratory. The plasma was separated in a refrigerated centrifuge at 4C, $2000 \mathrm{rpm}$ for 12 minutes, and then stored at $-80 \mathrm{C}$ until analyzed. Blood samples from the aorta were selected to represent the average concentration of cytokines from the whole body because regional differences in cytokine concentrations may occur throughout the body.

For BNP, $10 \mathrm{~mL}$ of blood was collected from a peripheral vein and plasma separated with the same procedure described above for cytokines. It was quantified using a rapid fluorescence immunoassay (Biosite Diagnostics Inc., San Diego, CA)..$^{21,22}$

The inflammatory cytokines tumor necrosis factor (TNF) $-\alpha$, interferon- $\gamma$, and transforming growth factor $-\beta$ were measured with commercially available enzyme-linked immunosorbent assay kits (R\&D Systems, Minneapolis, $\mathrm{MN})$. All tests were done in duplicate and the mean used as the result.

Statistical Analysis. Statistical analysis was done using SPSS 9 software (SPSS Inc., Chicago, IL). All data are 
expressed as mean \pm SEM. To compare continuous dependent variables, we used the Friedman test or Wilcoxon test. Correlations of BNP levels with demographic characteristics and other clinical and biochemical variables were performed using the Spearman test. To test for differences between independent variables, we used the MannWhitney or Kruskal-Wallis test. All comparisons were 2-tailed. Significant differences were said to exist at $P<.05$ for all parameters. Trends were said to exist at $\mathrm{P}<.1$ for exploratory reasons.

\section{Results}

Baseline Characteristics of the Study Population. Table I and Table II summarize the demographics, treatment medications, and clinical and biochemical assessment parameters of the patients with heart failure $(n=28)$ and the normal volunteers $(n=8)$. As shown, the mean age and body mass index for the heart failure group were similar to those of the control group. All heart failure patients had stable New York Heart Association (NYHA) class I to IV and were on standard 3-drug therapy, including angiotensinconverting enzyme inhibitors (89\%) or angiotensin receptor blockers (11\%), digoxin (85\%), spironolactone (79\%), and diuretics (93\%). The use of -adrenergic blocking agents in patients with Chagas disease heart failure has not been routinely recommended because of a lack of clinical data in this specific population; therefore, only $5(18 \%)$ of the heart failure patients were taking a $\beta$-blocker. There were no significant differences in the baseline heart rate and mean blood pressure level between patients with heart failure and controls. However, the mean LVEF, left ventricle diastolic diameter, and serum sodium level were significantly different in the heart failure patients compared with the control group $(P<.001)$.

Circulating Levels of BNP in Patients and Controls. The mean BNP levels were significantly higher in patients with heart failure $(568 \pm 87 \mathrm{pg} /$

Table II. Clinical and Biochemical Assessment Parameters of Studied Population

\begin{tabular}{|c|c|c|c|}
\hline & $\begin{array}{l}\text { Heart Fallure } \\
(N=28)\end{array}$ & $\begin{array}{l}\text { CONTROL } \\
(N=8)\end{array}$ & $P$ Value \\
\hline NYHA class, no. (\%) & $4(14.3)$ & - & - \\
\hline \multicolumn{4}{|l|}{ I } \\
\hline ॥ & $7(25)$ & - & - \\
\hline III & $11(39.3)$ & - & - \\
\hline IV & $6(21.4)$ & - & - \\
\hline QOL & $59.1 \pm 5.4$ & - & - \\
\hline 6-minute, $\mathrm{m}$ & $398 \pm 20$ & - & - \\
\hline LVEF, \% & $21.9 \pm 1.3$ & $63.1 \pm 1.2$ & $<.001$ \\
\hline LVEDD, mm & $67.1 \pm 1.4$ & $48.9 \pm 0.8$ & $<.001$ \\
\hline $\mathrm{RAP}, \mathrm{mm} \mathrm{Hg}$ & $9.3 \pm 1.1$ & $8.1 \pm 0.9$ & .79 \\
\hline MPAP, mm Hg & $25.8 \pm 2.0$ & $19 \pm 1.2$ & .018 \\
\hline PAPd, $\mathrm{mm} \mathrm{Hg}$ & $17.4 \pm 1.6$ & $10.4 \pm 0.9$ & .006 \\
\hline PCWP, mm Hg & $17 \pm 1.6$ & $9.6 \pm 0.65$ & .001 \\
\hline Serum sodium, mEq/L & $133.6 \pm 2.1$ & $142 \pm 3.1$ & $<.01$ \\
\hline TNF- $\alpha, p g / m L$ & $138 \pm 20$ & $4.4 \pm 2.5$ & $<.0001$ \\
\hline IFN- $\gamma, \mathrm{pg} / \mathrm{mL}$ & $266 \pm 62$ & $12 \pm 4.6$ & $<.01$ \\
\hline TGF- $\beta, p g / m L$ & $101 \pm 8.4$ & $114 \pm 8.5$ & .246 \\
\hline \multicolumn{4}{|c|}{$\begin{array}{l}\text { Abbreviations: IFN, interferon; LVEDD, left ventricle end diastolic diameter; LVEF, left } \\
\text { ventricle ejection fraction; MPAP, mean pulmonary artery pressure; NYHA, New York Heart } \\
\text { Association; PAPd, pulmonary artery pressure - diastolic; PCWP, pulmonary capillary } \\
\text { wedge pressure; QOL, quality of life (Minnesota Living With Heart Failure Questionnaire } \\
\text { score); RAP, right atrial pressure; 6-minute, distance walked in } 6 \text { minutes; TGF, transforming } \\
\text { growth factor; TNF, tumor necrosis factor. Values presented as mean } \pm \text { SE. }\end{array}$} \\
\hline
\end{tabular}

$\mathrm{mL})$ than in controls $(7.8 \pm 1.9 \mathrm{pg} / \mathrm{mL})$ $(P<.0001$; Figure 1). Even asymptomatic heart failure patients had higher BNP levels $(142 \pm 60 \mathrm{pg} / \mathrm{mL})$ than did normal controls $(P=.006)$.

Correlations of BNP Levels With Clinical, Biochemical, and Immunologic Variables. BNP levels correlated with increasing severity of symptoms, as reflected by increasing levels in NYHA functional class $(r=0.58 ; P=.001$; Figure 2). Patients with systemic congestion, as evidenced by the presence of peripheral edema and/or ascites, had higher BNP levels $(872 \pm 127 \mathrm{pg} / \mathrm{mL})$ than those who were euvolemic ( $424 \pm 98 \mathrm{pg} / \mathrm{mL} ; P=.012)$. The presence of a third heart sound was associated with higher levels of BNP $(912 \pm 105$ vs $358 \pm 105 \mathrm{pg} / \mathrm{mL}$; $P=.005)$.

Serum sodium level had a significant negative correlation with BNP level $(r=-0.66 ; \quad P<.002)$. Hyponatremic patients (sodium level $<135 \mathrm{mEq} / \mathrm{L}$ ) had significantly higher levels of BNP $(823 \pm 142$ vs $143 \pm 67 ; P=.002)$ compared with patients with normal serum sodium levels. Renal function, evaluated by urea levels, had a positive correlation $(r=0.51 ; P=.02)$ with BNP level. TNF- $\alpha$ was the only measured cytokine that had a positive correlation with BNP level $(r=0.46$; $P=.015$; Figure 3 ).

The presence of anemia (defined as a hemoglobin level $<12 \mathrm{mg} / \mathrm{dL}$ ) was associated with higher levels of BNP $(778 \pm 123$ for anemic vs $409 \pm 96 \mathrm{pg} /$ $\mathrm{mL}$ for non-anemic patients; $P=.032$; $r=-0.50 ; P=.009$ ).

Correlations of BNP Levels With Hemodynamics, Systolic and Diastolic Function. From the invasive hemodynamic measurements, mean systemic arterial pressure was inversely correlated with BNP level $(r=-0.55$; $P=.025)$. BNP levels had the strongest correlation with right atrial pressure $(r=0.80 ; P<.0001)$, followed by pulmonary capillary wedge pressure $(r=0.73 ; P<.0001)$. They were inversely correlated with the cardiac output $(r=-0.45 ; P=.036)$. The level of BNP 


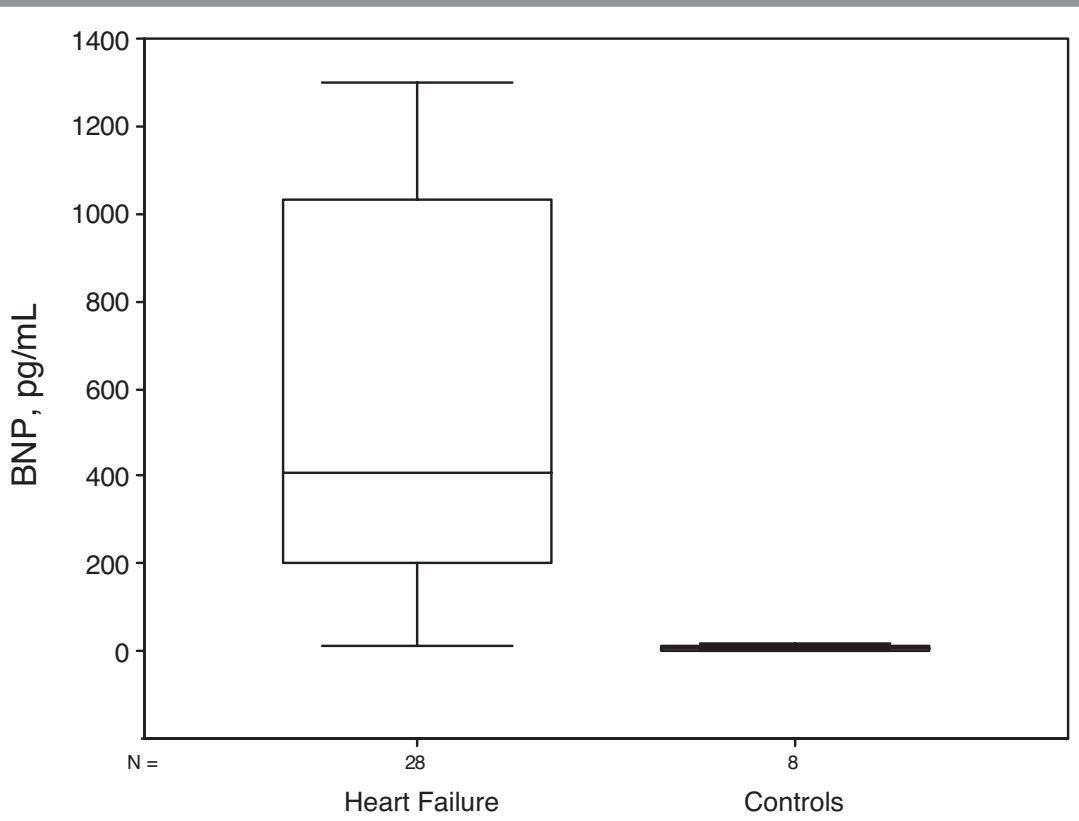

Group

Figure 1. B-type natriuretic peptide (BNP) concentrations in heart failure and control groups. $P<.001$.

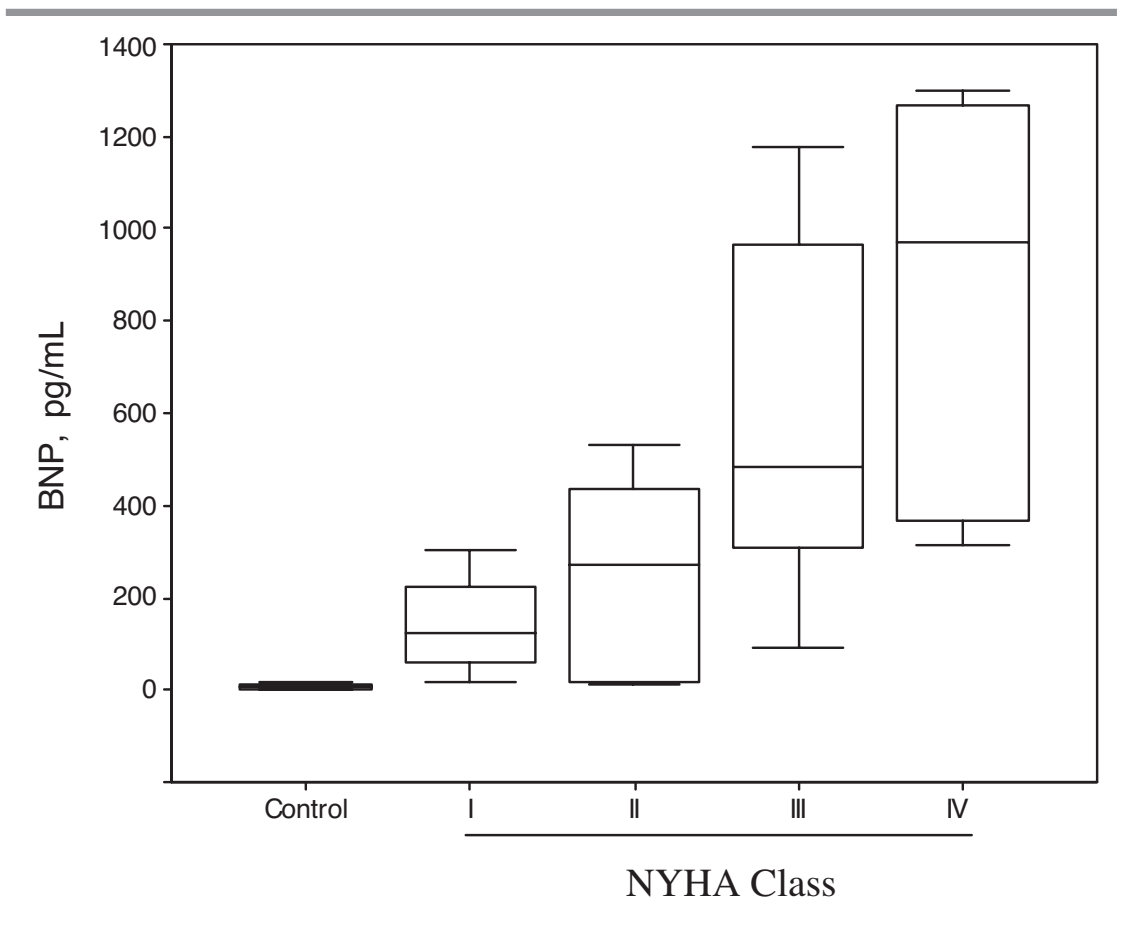

Figure 2. B-type natriuretic peptide (BNP) concentrations in control group and heart failure patients according to New York Heart Association (NYHA) functional class. $P=.006$ control vs NYHA class $I ; P=.027$ for comparisons between classes. had a significant, inverse correlation with systolic and diastolic ventricular function evaluated by LVEF ( $r=-0.48$; $P=.01)$ and isovolumetric relaxation time $(r=-0.54 ; P=.02)$.

Correlations With Exercise Capacity and Quality of Life Score. BNP levels correlated inversely with the distance walked in the 6-minute walking test $(r=-0.40 ; P=.032)$. There was no significant correlation with quality of life evaluated by the Minnesota questionnaire.

\section{Discussion}

This observational study investigated BNP levels in patients with heart failure due to Chagas cardiomyopathy. Our study is in accordance with previous observations that indicate that BNP levels correlate with both systolic and diastolic left ventricle dysfunction. ${ }^{23-25}$

Chagas disease heart failure is a model of heart failure that involves both ventricles simultaneously. ${ }^{26}$ Our findings extend the conclusions of observational studies in which the measurement of BNP levels was compared between normal individuals and patients with Chagas disease at different stages. ${ }^{14,15,27}$ After disease severity is accounted for, differences between patients with heart failure and controls were much more significant. We have also demonstrated that even patients with asymptomatic left ventricle dysfunction had higher BNP levels than normal controls. This finding is not common in other forms of heart failure, in which BNP levels are lower in the absence of congestion. ${ }^{22}$ We were able to demonstrate that BNP levels correlated with diastolic function. This finding is in accordance with what has been described previously in other forms of heart failure. ${ }^{23}$

This is the first study to correlate BNP levels with invasive hemodynamics in Chagas disease heart failure. Both the right and left sides of the heart were studied, and correlations were established with the hemodynamic profile. We were able 
to demonstrate that BNP levels correlate with both right and left heart parameters of ventricular dysfunction, with similar strength. This is an interesting finding and may represent a distinct characteristic of Chagas disease heart failure. Ischemic and hypertensive models of heart failure affect mainly the left ventricles, so one would expect that, in this setting, right heart correlations become weaker although still present. ${ }^{28}$ In Chagas disease heart failure, however, the heart is affected throughout, and the finding of strong correlations of BNP levels with right atrial and pulmonary wedge pressures is emblematic of this unique situation. In fact, recent evidence indicates that BNP levels predict survival better than levels of atrial natriuretic peptide. ${ }^{29} \mathrm{BNP}$ levels had an inverse relation to blood pressure level, which is expected because hypotension is also a marker of advanced heart failure and is particularly common in Chagas disease. As expected, BNP level was associated with the presence of clinical evidence of ventricular dysfunction and congestion, such as the presence of a third heart sound and systemic congestion. This is in accordance with previous observations in patients with Chagas disease, in whom the BNP level was more accurate in the identification of patients with ventricular dysfunction. ${ }^{30}$

The negative correlation of BNP levels with renal function is likely related to worsening heart failure and development of the cardiorenal syndrome rather than to accumulation of BNP due to diminished renal clearance. Serum sodium level had a negative correlation with BNP levels. This is an expected finding because hyponatremia is a well-recognized marker of poor prognosis in heart failure.

An interesting observation relates to the association of anemia with higher levels of BNP. The presence of anemia has been progressively recognized as a marker of worse prognosis in heart failure. $^{31}$

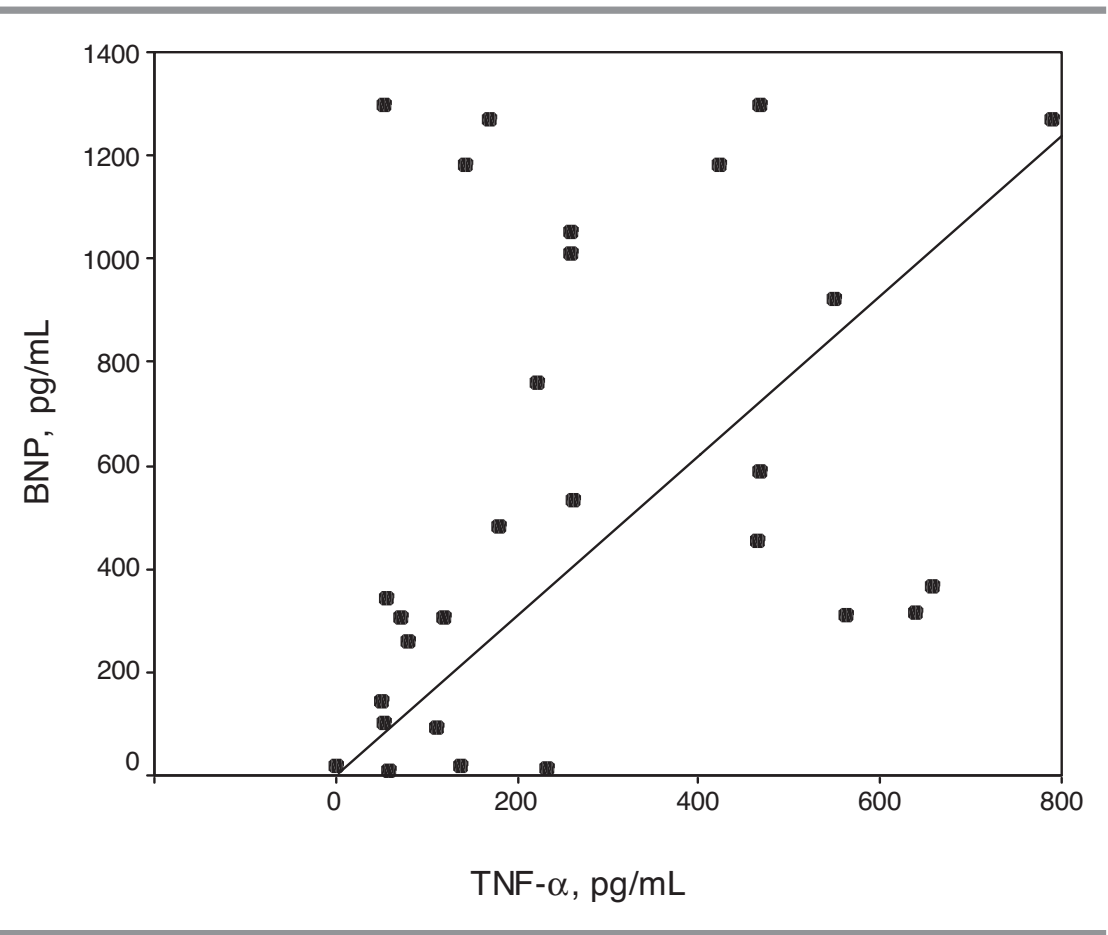

Figure 3. Correlations between B-type natriuretic peptide (BNP) and tumor necrosis factor $(\mathrm{TNF})-\alpha$ levels $(r=0.46 ; P=.015)$.

A particular strength of our study is that we could correlate BNP levels with immune activation in Chagas disease heart failure (Figure 3). In this particular form of disease, proinflammatory cytokines are up-regulated and are a key element in disease progression. ${ }^{4,32}$ BNP level correlated with the level of TNF- $\alpha$, one of the most potent cytokines involved in the pathogenic process of Chagas disease heart failure. This may represent a simple association of 2 independent prognostic variables, but it may also result from common regulatory processes and/or share similar genes. This association may also have therapeutic implications, regarding the development of new therapies targeted to decrease TNF- $\alpha$ levels. ${ }^{33}$ BNP may also be used as an indirect predictor of immune activation status.

The finding of an inverse correlation between BNP level and functional capacity is noteworthy and is in accordance with other studies. ${ }^{34}$ The 6 -minute walking test is a measure of submaximal exercise that correlates with daily activities. This correlation means that we can trust BNP levels to infer exercise capacity, ie, the higher the BNP level, the lower the exercise capacity.

We were unable to establish a correlation between BNP level and quality of life score, as assessed by the Minnesota Living With Heart Failure Questionnaire. This observation indicates that factors other than congestion are responsible for determining the complex nature of quality of life assessments.

\section{Conclusions}

We found that BNP levels in patients with Chagas disease heart failure are elevated and are a marker of disease severity that can be used to noninvasively evaluate and better risk stratify patients with this particular form of heart failure.

Acknowledgments: The authors wish to acknowledge the contribution of 
Dr. Alain Maisel from the Division of Cardiology and the Department of Medicine, Veteran's Affairs Medical Center and University of California,
San Diego, CA, for reviewing this document and Drs Augusto Almeida, Marco Sales and Eduardo Darze for performing the echocardiographic assessments. The technical assistance of Ana Gabriela Silva, Cristiane Carvalho, and Cláudio Brandão is gratefully acknowledged.

\section{ReFERENCES}

1 Redfield MM. Heart failure - an epidemic of uncertain proportions. $N$ Engl J Med. 2002;347: 1442-1444.

2 Dias JC, Silveira AC, Schofield CJ. The impact of Chagas' disease control in Latin America: a review Mem Inst Oswaldo Cruz. 2002;97(5):603-612.

3 Shulman IA, Appleman MD, Saxena S, et al. Specific antibodies to Trypanosoma cruzi among blood donors in Los Angeles, California. Transfusion. 1997;37:727-731.

4 Pontes-de-Carvalho L, Santana CC, Soares $M B$, et al. Experimental chronic Chagas' disease myocarditis is an autoimmune disease preventable by induction of immunological tolerance to myocardial antigens. J Autoimmun. 2002; 18(2):131-138.

5 Cunha-Neto E, Duranti M, Gruber A, et al. Autoimmunity in Chagas disease cardiopathy: biological relevance of a cardiac myosinspecific epitope crossreactive to an immunodominant Trypanosoma cruzi antigen. Proc Natl Acad Sci U S A. 1995:92:3541-3545.

6 Rassi A Jr, Rassi A, Little WC, et al. Development and validation of a risk score for predicting death in Chagas' heart disease. N Engl J Med. 2006;355:799-808.

7 Garcia S, Ramos CO, Senra JF, et al. Treatment with benznidazole during the chronic phase of experimental Chagas' disease decreases cardiac alterations. Antimicrob Agents Chemother. 2005;49:1521-1528.

8 Viotti R, Vigliano C, Lococo B, et al. Longterm cardiac outcomes of treating chronic Chagas disease with benznidazole versus no treatment. A nonrandomized trial. Ann Intern Med. 2006; 144:724-734.

9 Maisel AS, Krishnaswamy P, Nowak RM, et al; Breathing Not Properly Multinational Study Investigators. Rapid measurement of B-type natriuretic peptide in the emergency diagnosis of heart failure. $N$ Engl J Med. 2002;347(3): 161-167.

10 Berger R, Huelsman M, Stecker K, et al. B-type natriuretic peptide predicts sudden death in patients with chronic heart failure. Circulation. 2002; 105:2391-2396.

11 Mueller C, Scholer A, Laule-Kilian K, et al. Use of B-type natriuretic peptide in the evaluation and management of acute dyspnea. N Engl J Med. 2004;350:647-654.

12 Chen HH, Burnett JC. Natriuretic peptides in the pathophysiology of congestive heart failure. Curr Cardiol Rep. 2000;2:198-205.
13 Villacorta H, Duarte A, Duarte NM, et al. The role of B-type natriuretic peptide in the diagnosis of congestive heart failure patients presenting to an emergency department with dyspnea. Arq Bras Cardiol. 2002;79:569-572.

14 Melo RB, Parente GB, Victor EG. Measurement of human brain natriuretic. peptide in patients with Chagas' disease. Arq Bras Cardiol. 2005;84: 137-140

15 Talvani A, Rocha MOC, Cogan J, et al. Brain natriuretic peptide and left ventricular dysfunction in chagasic cardiomyopathy. Mem Inst Oswaldo Cruz. 2004;99:645-649.

16 Schiller NB, Shah PM, Crawford $M$, et al. Recommendations for quantitation of the left ventricle by two-dimensional echocardiography. American Society of Echocardiography Committee on Standards, Subcommittee on Quantitation of Two-Dimensional Echocardiograms. J Am Soc Echocardiogr. 1989;2:358-367

17 Stewart WJ, Rodkey SM, Gunawardena S, et al. Left ventricular volume calculation with integrated backscatter from echocardiography. J Am Soc Echocardiogr. 1993;6:553-563.

18 Rector TS, Kubo SH, Cohn JN. Patients' selfassessment of their congestive heart failure. Part 2: content, reliability and validity of a new measure, the Minnesota Living with Heart Failure Questionnaire. Heart Failure. 1987;3:198-209

19 Bittner $\mathrm{V}$, Weiner DH, Yusuf S, et al. Prediction of mortality and morbidity with a 6-minute walk test in patients with left ventricular dysfunction. JAMA. 1993;270:1702-1707.

20 Guyatt GH, Sullivan M, Thompson PJ, et al. The six minute walk: a new measure of exercise capacity in patients with chronic heart failure. Can Med Assoc J. 1985; 132:919-923.

21 Cheng V, Kazanegra R, Garcia A, et al. A rapid bedside test for B-type peptide predicts treatment outcomes in patients admitted for decompensated heart failure: a pilot study. J Am Coll Cardiol. 2001;37:386-391.

22 McCullough PA, Nowak RM, McCord J, ef al. B-type natriuretic peptide and clinical judgment in emergency diagnosis of hear failure: analysis from Breathing Not Properly (BNP) Multinational Study. Circulation. 2002; 106:416-422.

23 Maisel AS, Koon J, Krishnaswamy $P$, et al. Utility of B-natriuretic peptide as a rapid, point-of-care test for screening patients undergoing echocardiography to determine left ventricular dysfunction. Am Heart J. 2001;141:367-374.

24 Davis M, Espiner E, Richards G, et al. Plasma brain natriuretic peptide in assessment of acute dyspnoea. Lancet. 1994;343(8895):440-444.

25 Ribeiro $A L$, dos Reis $A M$, Barros $M V$, et al. Brain natriuretic peptide and left ventricular dysfunction in Chagas' disease. Lancet 2002;360:461-462

26 Rassi A Jr, Rassi A, Little WC. Chagas' heart disease. Clin Cardiol. 2000;23:883-889.

27 Marques DS, Canesin MF, BaruttaJ\&uacute; nior $\mathrm{F}$, et al. Evaluation of asymptomatic patients with chronic Chagas disease through ambulatory electrocardiogram, echocardiogram and B-type natriuretic peptide analyses. Arq Bras Cardiol. 2006;87:336-343.

28 Mariano-Goulart D, Eberle MC, Boudousq $\mathrm{V}$, et al. Major increase in brain natriuretic peptide indicates right ventricular systolic dysfunction in patients with heart failure. Eur J Heart Fail. 2003:5:481-488.

29 Heringer-Walther S, Moreira MC, Wessel $\mathrm{N}$, et al. Brain natriuretic peptide predicts survival in Chagas' disease more effectively than atrial natriuretic peptide. Heart. 2005;91 (3):385-387.

30 Ribeiro AL, Teixeira MM, Reis AM, et al. Brain natriuretic peptide based strategy to detect left ventricular dysfunction in Chagas disease: a comparison with the conventional approach. Int J Cardiol. 2006; 109(1):34-40.

31 Tepper D. Frontiers in congestive heart failure: anemia is associated with worse symptoms, greater impairment in functional capacity, and a significant increase in mortality in patients with advanced heart failure. Congest Heart Fail. 2002;8:235-236.

32 Mocelin AO, Issa VS, Bacal F, et al. The influ ence of aetiology on inflammatory and neurohumoral activation in patients with severe heart failure: a prospective study comparing Chagas' heart disease and idiopathic dilated cardiomyopathy. Eur J Heart Fail. $2005 ; 7(5): 869-873$

33 Pinto Dias JC. The treatment of Chagas disease (South American trypanosomiasis). Ann Intern Med. 2006;144(10):772-774.

34 Wieczorek SJ, Hager D, Barry MB, et al. Correlation of B-type natriuretic peptide level to 6-min walk test performance in patients with left ventricular systolic dysfunction. Clin Chim Acta. 2003;328:87-90. 